\title{
APLICAÇÃO DO TESTE DE CARGA PONTUAL MODIFICADO PARA SELEÇÃO DE BRITADORES PARA MINÉRIO DE FERRO
}

\author{
J. J. VARELA, V. RAAZ, D. B. MAZZINGHY*e J. F. C. RUSSO \\ ThyssenKrupp e Anglo American \\ douglas.mazzinghy@angloamerican.com*
}

Artigo submetido em novembro/2015 e aceito em novembro/2015

DOI: 10.15628/holos.2015.3665

\section{RESUMO}

A resistência à compressão uniaxial (UCS) é a propriedade mecânica mais aceita para dimensionamento de britadores destinados ao processamento de rochas e minérios. O teste de carga pontual (PLT) é o ensaio comumente utilizado para determinação do índice de resistência da rocha ou minério e pode ser utilizado para a determinação indireta da resistência à compressão. Neste contexto, realizou-se uma campanha de testes com diferentes litologias para o projeto Minas-Rio, localizado em Minas Gerais, utilizando a metodologia de teste de carga pontual modificada (MPLT) desenvolvida pela ThyssenKrupp. Esta metodologia correlaciona em um gráfico logarítmico a área da amostra com a força de ruptura e, a partir de uma análise estatística, define-se um intervalo de confiança para os valores medidos. Os resultados obtidos nesta campanha de testes indicaram a possibilidade de uso de britadores de rolos para as litologias estudadas.

PALAVRAS-CHAVE: Teste de carga pontual, resistência à compressão, britagem, Itabirito, Projeto Minas-Rio.

\section{APPLICATION OF MODIFIED POINT LOAD TEST TO SELECT CRUSHERS FOR IRON ORE}

\section{ABSTRACT}

The uniaxial compressive strength (UCS) is the mechanical property more acceptable for crusher design to processing rocks and ores. The point load test (PLT) is commonly used to determine the rock or ore resistance and can be used to indirect determination of the compressive strength. In this context, a test campaign was performed with different lithologies for the MinasRio project, located in Minas Gerais State, using the modified point load testing methodology (MPLT) developed by ThyssenKrupp. This methodology correlates in a logarithmic plot the area of the sample with the rupture strength and, from a statistical analysis, a confidence interval for the measured values is defined. The results of this test campaign indicated the possibility of using rolls crushers for the lithologies studied.

KEYWORDS: Point load test, compressive strength, crushing, itabirite, Minas-Rio Project. 


\section{INTRODUÇÃO}

A caracterização dos minérios de ferro geralmente está associada à sua composição química, mineralógica, estrutural e à sua origem geológica. Raramente encontram-se dados relacionados à resistência mecânica das rochas que compõem estes depósitos (Varela, 2013). Em geral, os minérios de ferro brasileiros podem ser classificados, basicamente, em hematitas e itabiritos. Os primeiros com teores de ferro superiores a $63,5 \%$ e os últimos, variando entre $30 \%$ e 63,5\%. Também se observa na literatura a adoção de inúmeras outras nomenclaturas para minérios de ferro em função da sua mineralogia, a saber: martíticos, goethíticos, especularíticos, magnetíticos, hematíticos, anfibolíticos, etc. Outro critério de classificação baseia-se nas propriedades físicas, diferenciando os minérios em compactos, friáveis e pulverulentos (Veríssimo, 1999 e Takehara, 2004).

O projeto Minas-Rio, de propriedade da empresa AngloAmerican, localizado no Munic'pio de Conceição do Mato Dentro, Estado de Minas Gerais, Região Sudeste do Brasil, iniciou suas atividades de produção no final de 2014. A Figura 1 apresenta a distribuição das litologias do depósito, excluindo os materiais estéril e canga.

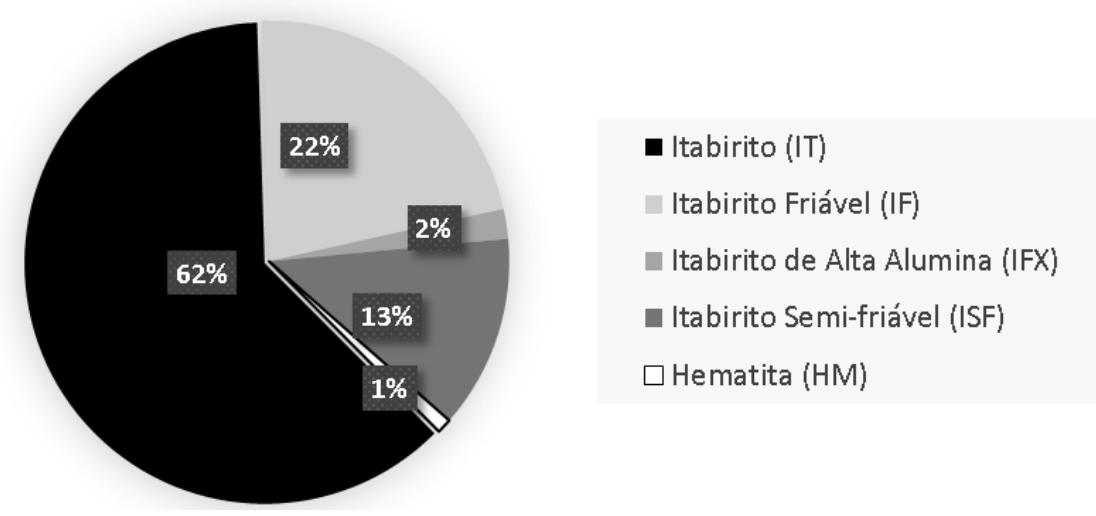

Figura 1: Distribuição das litologias do depósito

A atual planta de processamento mineral foi projetada para processar a litologia friável (IF), material de maior teor de ferro. Uma parte considerável da reserva do Minas-Rio é composta de minérios classificados como itabirito (IT). Este material difere do friável principalmente pelo menor teor de ferro, distribuição granulométrica mais grosseira e pelo menor consumo específico de energia na etapa de moagem primária (Mazzinghy et al., 2014).

O objetivo deste trabalho é verificar a possibilidade de aplicação de britadores de rolos para processamento futuro da litologia itabirito (IT), litologia de maior proporção no depósito, conforme ilustrado na figura 1. Uma campanha de testes com amostras provenientes de diferentes regiões do depósito foi realizada para fornecer informações sobre a dureza dos distintos litotipos.

\section{TESTE DE CARGA PONTUAL}

O teste de carga pontual (PLT = point load test) tem como resultado a determinação do índice de resistência de uma amostra padronizada em $50 \mathrm{~mm}$ de diâmetro $\left(I_{550}\right)$ a partir do qual pode-se calcular a resistência à compressão uniaxial (UCS = uniaxial compressive strength) do 
material. Atualmente, o UCS é o índice mais aceito para caracterizar a resistência à compressão de uma rocha. O PLT pode ser utilizado como uma medida indireta para determinação da resistência à compressão de uma rocha. Porém o PLT é um ensaio destrutivo, motivo pelo qual, o teste necessita ser repetido em inúmeros fragmentos do mesmo tipo de amostra, realizando-se uma análise estatística dos resultados. A Sociedade Internacional de Mecânica de Rochas (ISRM, 1985) define a metodologia utilizada para a realização do PLT. O PLT, por ser um teste de rápida e fácil execução, pode ser realizado tanto em laboratório quanto em campo, com amostras frescas de formato irregular, próximo ao local de ocorrência de um determinado minério, sem a necessidade de preparação de corpos de prova. Inúmeros autores têm apresentado correlações entre o IS50 e UCS. A generalização destas fórmulas para diferentes tipos de rochas pode resultar em grandes erros, especialmente para rochas que apresentam anisopropia (JahanGer e Ahmed, 2013, AkrameBakar, 2007, Rusnake Mark, 2015). Pinto (2013) ao caracterizar amostras de minério de ferro da região norte do Brasil através do método PLT comentou sobre a variação dos resultados de UCS ao aplicar-se as relações disponíveis na literatura.

O teste de carga pontual modificado (MPLT; do inglês modified point load test) é uma evolução do PLT. A metodologia MPLT correlaciona em um gráfico logarítmico a área da amostra com a força de ruptura. A partir de um uma análise estatística define-se um intervalo de confiança para os valores medidos. Desde a década de 90, a ThyssenKrupp tem aplicado o MPLT para selecionar equipamentos de mina (escavadeiras de rodas de caçamba), de processo (britadores) e para a definição dos critérios de projeto destas máquinas (Unland e Raaz, 1998). A Figura 2 apresenta os modelos de equipamentos desenvolvidos pela Thyssenkrupp para a execução do MPLT.
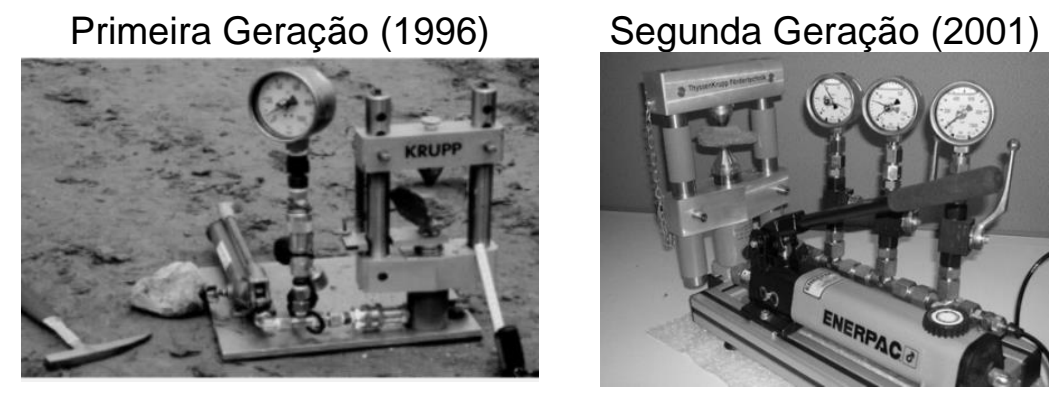

Terceira Geração (2013)

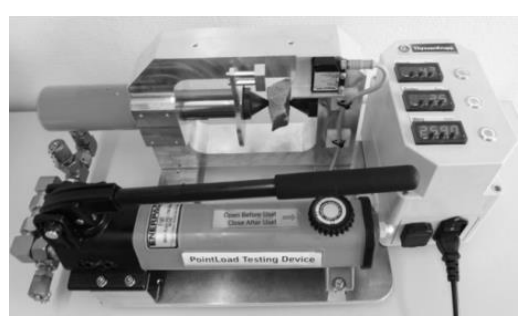

Figura 1- Equipamentos desenvolvidos para execução de MPLT

A Figura 3 exemplifica a metodologia MPLT, sendo que os valores da área da seção do fragmento de rocha, representada pelo diâmetro equivalente $\left(D_{e}\right)$, em escala logarítmica, estão correlacionados com a força de ruptura (valores máximos suportados pela amostra), ver equação 1. Neste gráfico também é apresentado o intervalo de confiança e a linha de regressão calculada a partir da dispersão dos dados medidos (inclinação). 


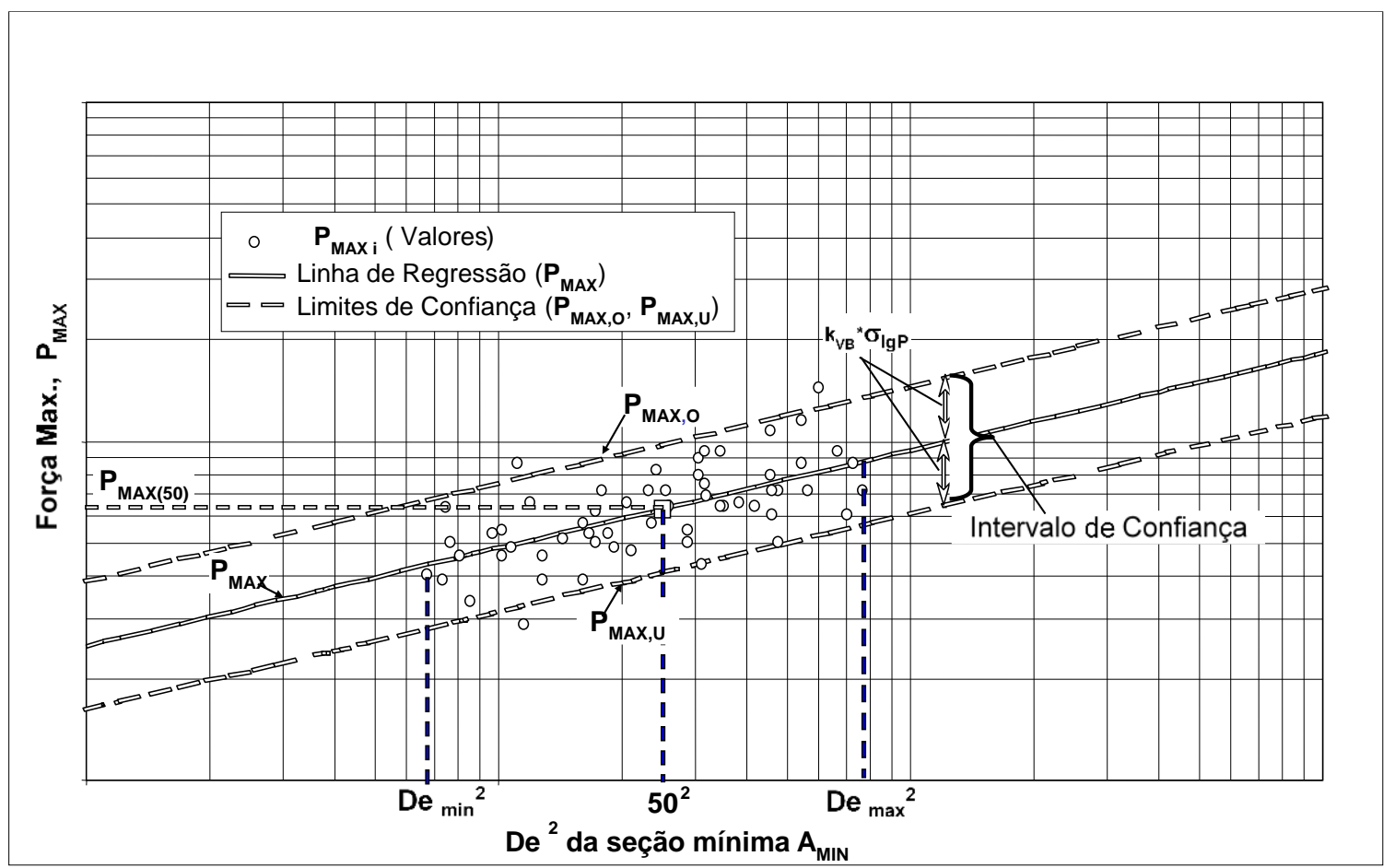

Figura 2 - Dados obtidos pelo MPLT apresentados em escala logarítmica (relação entre a seção mínima e a força máxima de ruptura)

$P_{\max (50)}=$ força de ruptura normalizada para o tamanho de $50 \mathrm{~mm}$

$P_{\max }=$ força de ruptura dependendo do tamanho (medido no manômetro do MPLT)

$D_{e}=$ diâmetro equivalente a área da seção do fragmento de rocha (medido na amostra)

$m=$ constante obtida pela linha de regressão (inclinação da reta obtida)

De acordo com a ISRM, o IS(50) pode ser determinado através da Equação 2.

$$
I_{\mathrm{S}(50)}=\frac{P_{\max (50)}}{50^{2}}
$$

A conversão do $I_{S(50)}$ para UCS pode ser determinada através das Equações 3 a 5 , dependendo do valor do $I_{S(50):}$

$$
\begin{array}{ll}
\text { se } I_{\mathrm{s}(50)} \leq 1, & U C S=13 \cdot I_{\mathrm{s}(50)} \\
\text { se } 1<I_{\mathrm{s}(50)} \leq 2, & U C S=13+18 \cdot\left(I_{\mathrm{s}(50)}-1\right) \\
\text { se } I_{\mathrm{s}(50)}>2, & U C S=31+24 .\left(I_{\mathrm{s}(50)}-2\right)
\end{array}
$$

\section{MATERIAIS E MÉTODOS}

A Figura 4 apresenta imagens das amostras extraídas a partir de furos de sondagens que foram utilizadas para execução do MPLT. Cada amostra foi composta de dez fragmentos de tamanhos similares para a realização de cinco ensaios na direção perpendicular e de cinco ensaios na direção paralela ao bandamento. A repetição de ensaios visa à obtenção de um valor médio e minimização do erro inerente ao teste. 


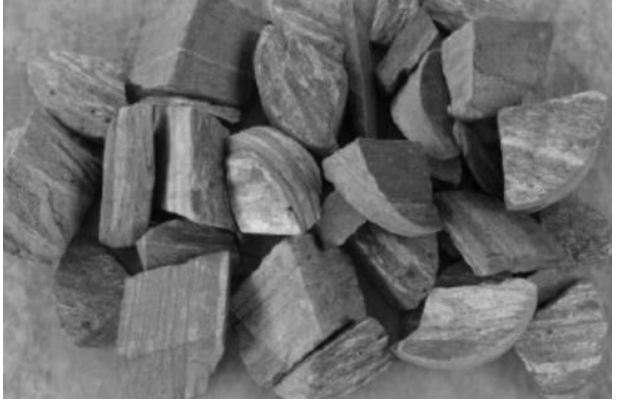

Itabirito

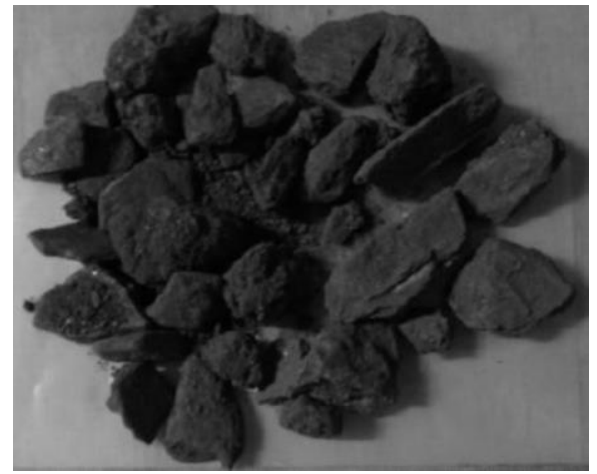

Canga

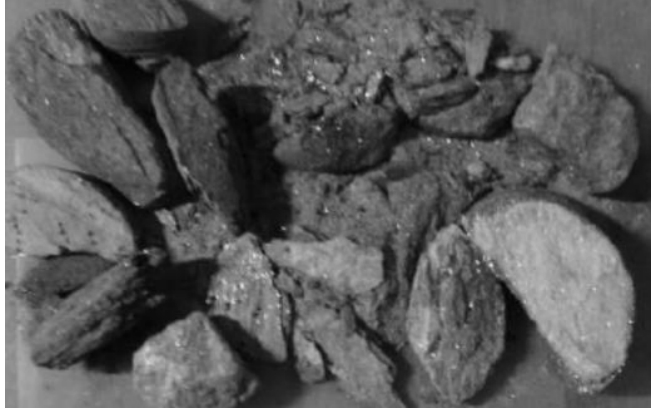

Semi-Friável

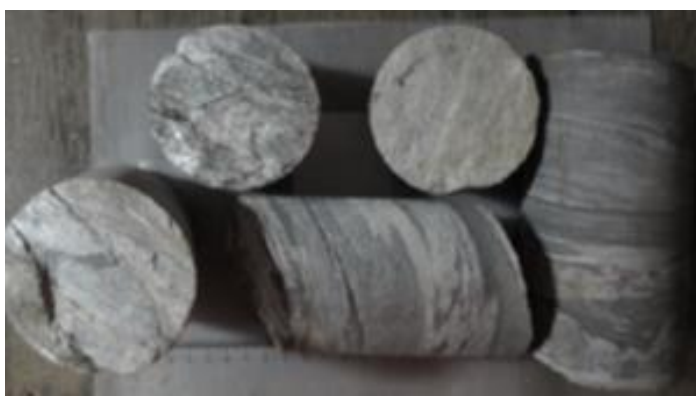

Quartizito

Figura 3- Litotipos testados

Foram testadas vinte e seis amostras de itabirito (IT), quatro amostras de itabirito semifriável (ISF), cinco amostras de quartzito e cinco amostras de canga. Eventualmente, estas duas últimas litologias, consideradas estéril, podem ser processadas, devido à diluição na lavra. Além disso, amostras de itabirito in-situ foram coletadas na mina. No total, foram realizados mais de quinhentos MPLT. Neste trabalho utilizou-se a segunda geração do equipamento MPLT desenvolvido pela ThyssenKrupp.

\section{RESULTADOS E DISCUSSÃO}

A Figura 5 apresenta os valores de resistência à compressão determinados para as amostras de itabirito. Neste gráfico, os resultados dos testes realizados nas direções perpendicular e paralela ao bandamento da rocha são apresentados juntamente com o valor médio. Os valores de resistência à compressão do itabirito (IT) podem ser considerados médios, entretanto verificou-se uma grande dispersão dos resultados sendo que a direção perpendicular à estratificação revela as maiores resistências. Neste sentido, as amostras de itabirito apresentam anisotropia relacionada ao seu bandamento (camadas intercaladas de minerais de ferro e quartzo). 


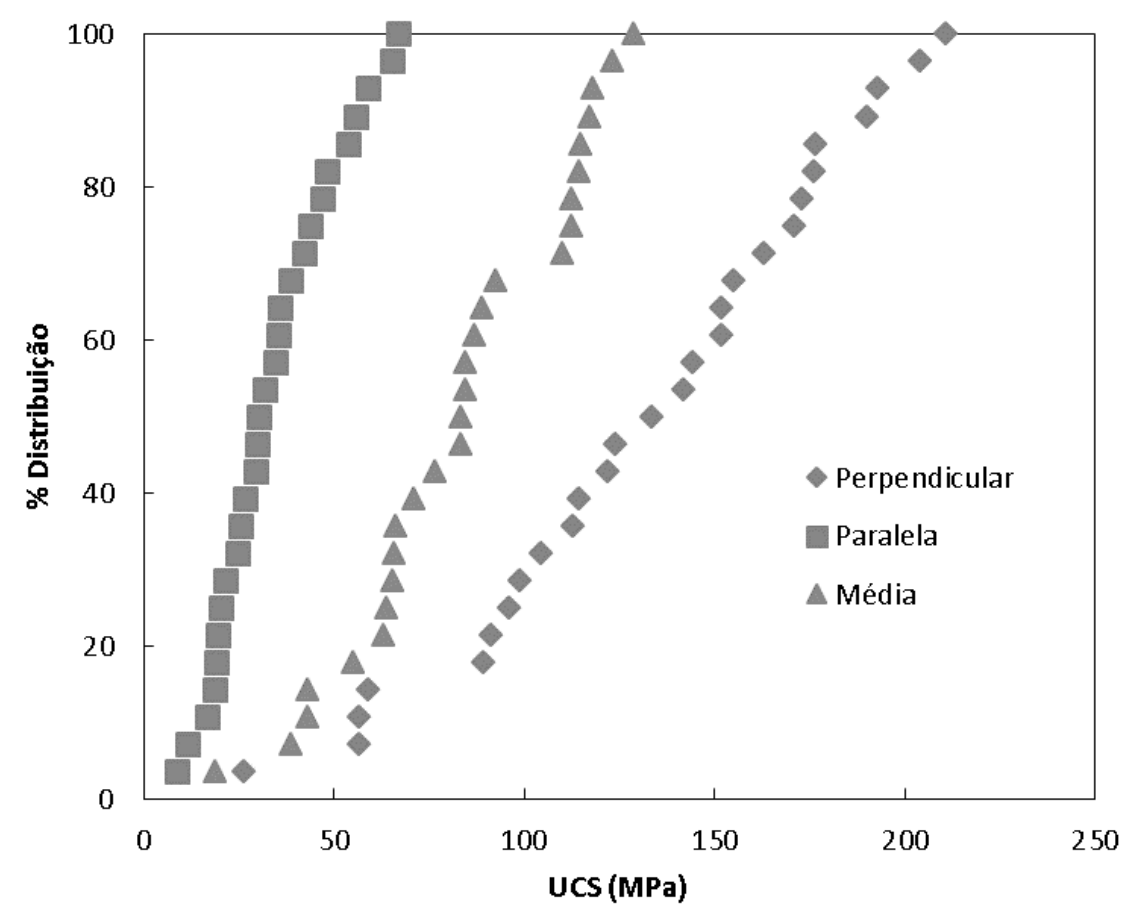

Figura 5 - Valores de resistência à compressão das amostras de Itabirito

A Figura 6 ilustra a tendência à geração de fragmentos tabulares/lamelares durante as operações de fragmentação da rocha itabirítica. $O$ itabirito apresentou um valor médio para o índice de resistência PLT $\left(I_{S(50)}\right)$ da ordem de 5,5, correspondendo a uma resistência média à compressão (UCS) de aproximadamente $120 \mathrm{MPa}$.

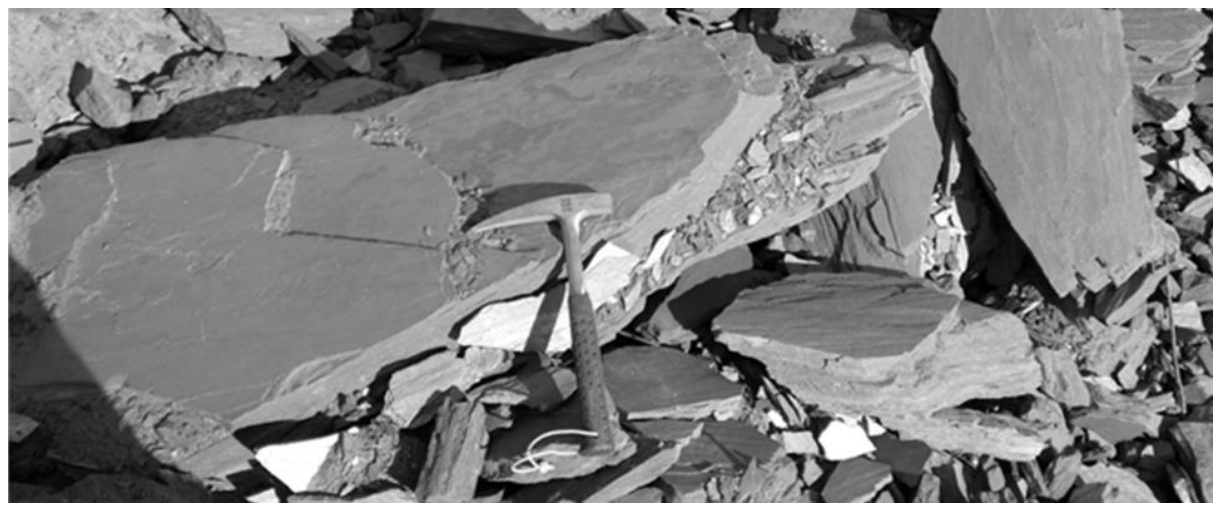

Figura 6 - Amostra in-situ de minério de ferro itabirítico apresentando um formato tabular/lamelar

O itabirito semi-friável apresentou valores de resistência à compressão baixos (média inferior a $50 \mathrm{MPa}$ ) e, na maioria das vezes, com aspectos de oxidação/degradação resultantes de fenômenos de intemperismo.

A litologia canga (material estéril) não apresentou anisotropia. A resistência à compressão desta rocha também é baixa.

A litologia quartzito (material estéril) apresentou uma ampla faixa de valores de resistência à compressão. Em alguns casos, observou-se fragmentos de quartzo de média a alta resistência (superior a $100 \mathrm{MPa}$ ) misturados com o minério de ferro e lentes arenosas friáveis (resistência à compressão inferior a $50 \mathrm{MPa}$ ). 
Uma criteriosa avaliação da resistência à compressão da rocha é fundamental para a seleção de um determinado tipo de britador e para a definição dos critérios de projeto do mesmo (Varela, 2011). Obviamente, a influência desta e de outras propriedades físico-químicas, tais como a abrasividade, densidade, teor etc., não se restringe à etapa de britagem, estendendo-se à outras etapas do processo, desde a classificação, moagem e etapas subsequentes de concentração.

A determinação destas propriedades das rochas que formam o depósito mineral (minério e estéril) também permite que as atividades de lavra sejam realizados de forma mais eficiente e econômica. No trabalho de Lima et al. (2013) a porosidade, o módulo de elasticidade e velocidade de propagação de onda são utilizadas para a diferenciação de litotipos e, consequentemente, melhoria nas atividades de perfuração. Desta forma é possível obter uma maior eficiência das operações unitárias de lavra e beneficiamento e consequentemente a maximização da recuperação do minério de ferro com a correta interpretação das características do material processado.

Sugere-se a inclusão da propriedade de resistência à compressão no banco de dados que compõe o modelo geometalúrgico da jazida e, por fim, a avaliação da sua influência na regulagem de toda a planta. Os resultados deste trabalho também permitem o desenvolvimento de um banco de dados e a sua análise comparativa com outros importantes depósitos mundiais de minério de ferro, como, por exemplo, os localizados no norte do Brasil (Carajás), sudoeste do Brasil (Quadrilátero Ferrífero), Centro-oeste (Corumbá), África, Austrália (Pilbara), China, Índia e EUA.

\section{CONCLUSÕES}

A determinação da resistência à compressão das rochas que formam o depósito mineral (minério e estéril) é fundamental para a seleção de britadores e definição dos seus parâmetros de projeto. O teste de carga pontual modificado aplicado no itabirito (IT) revelou valores de resistência à compressão média (120 MPa), contudo, verificou-se uma grande dispersão dos resultados sendo que a direção perpendicular à estratificação revela as maiores resistências. Por outro lado, foram obtidos valores baixos de resistência à compressão para as litologias canga e quartzito (estéril), exceto quando testados alguns fragmentos de quartzo associados a veios de maior compacidade. A partir dos resultados obtidos neste trabalho vislumbra-se um enorme potencial para adoção de britadores de rolos de maior eficiência e produtividade, quando comparados com britadores de mandíbulas, na etapa de britagem primária do Projeto Minas-Rio.

\section{AGRADECIMENTOS}

Os autores agradecem à ThyssenKrupp e à AngloAmerican por autorizar a publicação dos dados constantes deste trabalho.

\section{REFERÊNCIAS BIBLIOGRÁFICAS}

1. Akram, M. and Bakar, M.Z.A., Correlation between Uniaxial Compressive Strength and Indirect Strength Indices of Rocks of Salt Range, Pak. J. Engg. \& Appl. Sci. Vol. 1, 8 p, 2007. 
2. ISRM, Suggested method for determining point load strength- ISRM Commission on Testing Methods, Working Group on Revision of the Point Load Test Method, Int. J. Rock Mech. Min.Sci. \&Geomech. Abstr., 22.,2, 51-60, 1985.

3. JahanGer, Z.K, Ahmed, A.A., Correlation Between Point Load Index And Very Low Uniaxial Compressive Strength Of Some Iraqi Rocks, Australian Journal of Basic and Applied Sciences, 7(7): 216-229, 2013.

4. Lima, P.H.A., Soares, J.A., Rosa, I.B.C., Carvalho, R.F., Braga, M.A., Silva, M.D., Petrofísica de Minério de Ferro, Copyright 2013, SBGf - Sociedade Brasileira de Geofísica, 13th International Congress of the Brazilian Geophysical Society, Rio de Janeiro, Brazil, 6 p, 2013.

5. Mazzinghy, D.B., Russo, J.C.F., Pimentel, D.A, Parâmetros cinéticos de moagem de itabiritos compacto e friável, HOLOS, Ano 30, Vol. 3 - Edição Especial - XXV ENTMME / VII MSHNT, 10 p., 2014.

6. Pinto, C. Determinação da resistência ao carregamento pontual point PointLoad Test, Escola de Engenharia, UFMG, 13 p., 2013.

7. Rusnak, J., and Mark, C., Using the point load test to determine the uniaxial strength of coal, Pesquisa Mineral [Internet] 2015; [citado 2015 Feb 20], 10 p. Disponível em: http://www.cdc.gov/niosh/mining/UserFiles/works/pdfs/utplt.pdf.

8. Takehara, L. Caracterização geometalúrgica dos principais tipos de minérios de ferro brasileiros na fração sínter feed. Tese de Doutorado, UFRGS, Porto Alegre, 403 p., 2004.

9. Unland G., Raaz V.: Die formale Charakterisierung der Gesteine - ein Beitrag aus der Sicht des Maschinenbaus. Z. geol. Wiss. 26 (3/4), 315-328, Berlin, 1998.

10. Varela, J.J. Critérios de seleção de britadores aplicados ao processamento mineral, Anais XXIV Encontro Nacional de Tratamento de Minérios e metalurgia Extrativa, Salvador-BA-Brasil, 8 p., 2011.

11. Varela, J.J. Desenvolvimento e aplicação de britadores primários para minério de ferro, Anais XXV Encontro Nacional de Tratamento de Minérios e Metalurgia Extrativa \& VIII Meeting ofthe Southern Hemisphereon Mineral Technology, Goiânia - GO, 8 p., 2013.

12. Veríssimo, C. U. V. Jazida de Alegria: Gênese e Tipologia dos Minérios de Ferro (Minas 3,4 e5) - Porção Ocidental. [Tese (Doutorado]. Pós- Graduação em Geociências/Geologia Regional IGCE-UNESP, 234 p., 1999. 